\title{
Magnetic Resonance Imaging of Bilateral Hands Is More Optimal Than MRI of Unilateral Hands for Rheumatoid Arthritis
}

\author{
Ying-Qian Mo, Ze-Hong Yang, Hai-Ning He, Jian-Da Ma, Jin-Jian Liang, Wei-Ke Zeng, \\ Guang-Zi Shi, Jun Shen, and Lie Dai
}

ABSTRACT. Objective. To explore the advantages of magnetic resonance imaging (MRI) of bilateral hands in
rheumatoid arthritis (RA).
Methods. Consecutive patients with active RA were recruited for clinical assessments, radiographs,
and MRI of bilateral hands. Bilateral hands were scanned simultaneously on $3.0 \mathrm{~T}$ whole-body MRI
system and were scored on synovitis, osteitis, and bone erosion according to the RA MRI scoring
(RAMRIS) system.

Results. Among 120 patients included, wrist bones and metacarpophalangeal joint (MCPJ) 2 proximal showed bone erosion in early RA. The second to fifth metacarpal bases and the second to fourth MCPJ distal showed more bone erosion in mid-stage or late-stage RA. When MRI of dominant unilateral hand was analyzed, MRI synovitis and osteitis in 5\% of wrists and 3 MRI features in 5-14\% of MCPJ were misdiagnosed (McNemar test, all $\mathrm{p}<0.05$ ). There were $46 \%$ wrist synovitis, $29-52 \% \mathrm{MCPJ}-5$ synovitis, $45 \%$ wrist osteitis, and 20\%-34\% MCPJ2-5 osteitis not detected by joint tenderness and/or swelling. When the clinically more severe hand was selected for MRI of unilateral hand according to physical examination, MRI synovitis in 5\% of wrists and 3 MRI features in 7-15\% of MCPJ were misdiagnosed (all $\mathrm{p}<0.05$ ). Scatter plots and linear regression analyses were used to illustrate RAMRIS between dominant or selected hand ( $\mathrm{Y}$ values) and nondominant or nonselected hand (X values). All linear models were markedly different from a $\mathrm{Y}=\mathrm{X}$ linear model, indicating the dominant or clinically more severe hand could not represent the contralateral hand to evaluate RAMRIS.

Conclusion. MRI of bilateral hands is more optimal than MRI of the unilateral hand in RA. (First Release May 1 2018; J Rheumatol 2018;45:895-904; doi:10.3899/jrheum.171044)

Key Indexing Terms:

ARTHRITIS RHEUMATOID ARTHRITIS MAGNETIC RESONANCE IMAGING RAMRIS

From the Departments of Rheumatology and Radiology, Sun Yat-Sen Memorial Hospital; Zhongshan School of Medicine, Sun Yat-Sen University, Guangzhou, China.

Supported by the National Natural Science Foundation of China (grant no. 81471597, 81671612, 81601427), Specialized Research Fund for the Doctoral Program of Higher Education (grant no. 20130171110075), the Natural Science Foundation of Guangdong Province (grant no. 2016A030313307, 2014A030313074, 2017A030313470), and the Yat-Sen Scholarship for Young Scientists.

Y.Q. Mo, MD, Department of Rheumatology, Sun Yat-Sen Memorial Hospital, Sun Yat-Sen University; Z.H. Yang, MD, Department of Radiology, Sun Yat-Sen Memorial Hospital, Sun Yat-Sen University; H.N. He, Zhongshan School of Medicine, Sun Yat-Sen University; J.D. Ma, MD, PhD, Department of Rheumatology, Sun Yat-Sen Memorial Hospital,

Sun Yat-Sen University; J.J. Liang, MD, Department of Rheumatology, Sun Yat-Sen Memorial Hospital, Sun Yat-Sen University; W.K. Zeng, MD, Department of Radiology, Sun Yat-Sen Memorial Hospital, Sun Yat-Sen University; G.Z. Shi, MD, Department of Radiology, Sun Yat-Sen Memorial Hospital, Sun Yat-Sen University; J. Shen, MD, PhD, Prof., Department of Radiology, Sun Yat-Sen Memorial Hospital, Sun Yat-Sen University; L. Dai, MD, PhD, Prof., Department of Rheumatology, Sun Yat-Sen Memorial Hospital, Sun Yat-Sen University. Drs. Mo and Yang contributed equally to this work.

Address correspondence to Prof. L. Dai, Department of Rheumatology, Sun Yat-Sen Memorial Hospital, Sun Yat-Sen University, 107 Yanjiang West Road, Guangzhou 510120, China.E-mail: dailie@mail.sysu.edu.cn Accepted for publication January 19, 2018.
Rheumatoid arthritis (RA) is a systemic autoimmune disease characterized by chronic inflammation in the synovium (synovitis) and bone (osteitis), leading to joint destruction and even deformity. Although conventional radiography is considered the gold standard for imaging articular structure, its sensitivity for RA diagnosis and disease measurement is low and it almost cannot reflect inflammation ${ }^{1}$. Magnetic resonance imaging (MRI), which has advantages in visualizing inflammation and detecting bone erosion without exposure to radiation, can show synovitis, osteitis, and bone erosion in patients with RA. Osteitis is a strong independent predictor for subsequent radiographic progression ${ }^{2}$. Only MRI, not radiography or ultrasound, can detect osteitis. Additionally, physical examination such as tender or swollen joint counts correlated poorly with MRI osteitis ${ }^{3}$.

MRI has been increasingly used in diagnosis, disease measurement, and treatment response for research and clinical purposes in $\mathrm{RA}^{4,5}$. A validated semiquantitative scoring methodology for MRI synovitis, osteitis, and bone erosion was developed by the Outcome Measures in Rheumatology Clinical Trials (OMERACT): the RA MRI

Personal non-commercial use only. The Journal of Rheumatology Copyright @ 2018 . All rights reserved. 
scoring (RAMRIS) system ${ }^{6,7,8,9}$. In 2003, RAMRIS was generated from databases consisting of images of dominant wrists and/or metacarpophalangeal joints (MCPJ); MRI evaluation of unilateral hands was then recommended by OMERACT $^{7,8}$. In the majority of the succeeding studies, RAMRIS was evaluated for the clinically more severe hand or dominant hand in patients with $\mathrm{RA}^{3,10-23}$.

RA is characterized by symmetrical joint involvement. Physical examination and radiographs are usually performed on bilateral hands of patients with RA. A study compared radiographs of unilateral and bilateral hands in view of lower cost and exposure to radiation and found that $24-40 \%$ of patients would be incorrectly classified as nonerosive when radiographs of unilateral hands were evaluated, and progression would be missed in $21 \%-31 \%$ of patients ${ }^{24}$. A report on a small cohort ${ }^{25}$ of patients with RA $(n=35)$ by low-field $(0.2 \mathrm{~T})$ extremity MRI in 2005 was the only published report comparing unilateral and bilateral hands by MRI, in which only bone erosion was evaluated because of lack of T2-weighted and contrast-enhanced images. This study showed that MRI of bilateral hands detected radiological progression in more patients with RA $(n=29)$ than MRI of unilateral hands did $(\mathrm{n}=25)$; however, the difference was not statistically significant ${ }^{25}$. More recently, high performance of whole-body MRI and multichannel synergic coils enabled bilateral hands to be scanned simultaneously; it makes scanning faster, more accurate, and more convenient than before. Herein we used 3.0 T whole-body MRI for patients with RA to explore the advantages of MRI of bilateral hands.

\section{MATERIALS AND METHODS}

Patients. Consecutive patients with RA who fulfilled the 1987 American College of Rheumatology (ACR) revised classification criteria for RA or the 2010 ACR/European League Against Rheumatism classification criteria for early RA, and who had active disease [28-joint count Disease Activity Score using C-reactive protein (DAS28-CRP) $\geq 2$.6] were recruited between April 2014 and April 2016 from Sun Yat-Sen Memorial Hospital, Sun Yat-Sen University, Guangzhou, China. Patients were excluded if they refused or had contraindications for MRI examinations or contrast agents. This study was conducted in compliance with the Helsinki Declaration. The Medical Ethics Committee of Sun Yat-sen Memorial Hospital approved the protocol (SYSEC-2009-06) and all patients signed written informed consent.

Clinical assessments and radiographic assessment. Demographic characteristics including sex, age, disease duration, and prior therapy before recruitment were recorded. Clinical assessments and radiographs of bilateral hands (anteroposterior view) were performed just before MRI examination, as described ${ }^{26,27}$. Radiographs were scored according to the modified total Sharp/van der Heijde score (mTSS) of the hands. Subjects with mTSS $>10$ were considered as having radiographic joint damage ${ }^{28}$.

MRI assessment. Bilateral hands of each patient were scanned simultaneously on 3.0 T whole-body MRI system with an 8-channel sense head coil (Achieva; Philips Medical Systems). Each patient was imaged in a prone position, with pronation of bilateral hands. Hand movement was avoided with the aid of sandbags on forearms. The imaging sequences comprised coronal turbo spin echo fat-suppressed T2-weighted imaging [repetition time (TR) $2718.2 \mathrm{~ms}$, echo time (TE) $30 \mathrm{~ms}$, slice thickness/gap 2.5/0 mm, field of view (FOV) $128 \times 128$, matrix $312 \times 312$ ], coronal spin echo T1-weighted imaging (TR $500 \mathrm{~ms}$, TE $15 \mathrm{~ms}$, slice thickness/gap 2.5/0 mm, FOV $128 \times 128$, matrix $356 \times 275)$, and axial turbo spin echo fat-suppressed T2-weighted imaging (TR $3443.7 \mathrm{~ms}$, TE $30 \mathrm{~ms}$, slice thickness/gap 5/2 mm, FOV $128 \times 128$, matrix $312 \times 310$ ). Contrast-enhanced imaging was initiated immediately after intravenous injection of $0.2 \mathrm{mmol} / \mathrm{kg}$ Gd-DTPA (Magnevist; Bayer Pharma AG), with imaging sequences of axial spin echo fat-suppressed T1-weighted imaging (TR $500 \mathrm{~ms}$, TE $15 \mathrm{~ms}$, slice thickness/gap 5/2 mm, FOV $128 \times 128$, matrix $190 \times 312$ ) and coronal spin echo fat-suppressed T1-weighted imaging (TR $500 \mathrm{~ms}$, TE $15 \mathrm{~ms}$, slice thickness/gap 2.5/0 mm, FOV $128 \times 128$, matrix $275 \times 356$ ).

All MRI images were assessed according to the scoring system of MRI synovitis, osteitis, and bone erosion, as indicated in the definitions and atlas (standardized reference images) of the OMERACT 2002 RAMRIS ${ }^{6,9,29,30}$ For synovitis, each hand was scored in MCPJ2-5 and 3 wrist regions, including distal radioulnar, radiocarpal, and intercarpal-carpometacarpal joints; the scale is $0-3$. For osteitis and bone erosion, each hand was scored in 15 wrist bones ( 8 carpal bones, distal radius, distal ulna, and 5 metacarpal bases), metacarpal heads (proximal), and phalangeal bases (distal) of MCPJ2-5. The scale is $0-3$ for osteitis and 0-10 for bone erosion. As a summary, unilateral hand synovitis score ranged from 0 to 21 , osteitis score from 0 to 69 , and bone erosion score from 0 to 230; bilateral hands synovitis score ranged from 0 to 42 , osteitis score from 0 to 138 , and bone erosion score from 0 to 460 .

All MRI images were scored by 2 experienced radiologists ( $\mathrm{ZHY}$ and GZS, with 7 and 3 years of experience in musculoskeletal MRI, respectively) who were blinded to the patients' clinical findings and the objectives of our study. Specifically, they did not know dominant hands would be compared to nondominant hands when assessing MRI images. Reliability and agreement were assessed using intraclass correlation coefficient (ICC). The mean ICC of interobserver agreement was 0.852 for synovitis score, 0.739 for osteitis score, and 0.815 for bone erosion score. The mean ICC of intraobserver agreement was 0.837 for synovitis score, 0.763 for osteitis score, and 0.930 for bone erosion score. All the above agreements were considered high (ICC 0.6-0.8) to very high (ICC $\geq 0.8)^{31}$

Statistical analysis. Statistical analyses were performed with SPSS for Windows 19.0 (SPSS Inc.). Data were presented as frequencies and percentages for categorical variables; or median (interquartile range; IQR) for continuous variables. Spearman rank (r) correlation analysis generated the correlation coefficient. Chi-squared test or Fisher's exact test was used for comparison of categorical variables between 2 independent groups. McNemar test was used for comparison of categorical variables between 2 paired groups. Mann-Whitney rank-sum test was used for comparison between 2 independent groups, and Kruskal-Wallis 1-way ANOVA on ranks among $\geq 3$ groups. Linear regression analysis was used to illustrate the relationship between RAMRIS in the dominant or selected hand and RAMRIS in the nondominant or nonselected hand. All significance tests were 2-tailed and were conducted at the $5 \%$ significance level.

\section{RESULTS}

Demographic characteristics of patients with RA. A total of 138 patients with RA were recruited. All patients finished MRI examinations of bilateral hands, and 18 of them were excluded because their MCPJ could not be imaged in a single coronal view of MRI for severe deformity. Thus 120 patients with RA were included for statistical analyses (Table 1). The median age was 52 years (range 24-79) and 79\% were female. There were $52 \%, 35 \%$, and $13 \%$ of patients, respectively, with high, moderate, and low disease activity according to DAS28-CRP. Bony erosions were detected in $97 \%$ of patients and radiographic joint damage in $79 \%$. One patient was left-handed and 119 patients were right-handed. Seventy patients $(58 \%)$ were treatment-naive and never took

Personal non-commercial use only. The Journal of Rheumatology Copyright (C) 2018. All rights reserved 
Table 1. Demographic and clinical characteristics of the 120 patients with RA. Values are n (\%) or median (IQR).

\begin{tabular}{|c|c|}
\hline Characteristics & Values \\
\hline Female & $95(79)$ \\
\hline Age, yrs & $52(44-61)$ \\
\hline Disease duration, mos & $48(12-120)$ \\
\hline \multicolumn{2}{|l|}{ Core disease activity indicators } \\
\hline 28-TJC & $9(3-15)$ \\
\hline 28-SJC & $4(2-9)$ \\
\hline PtGA & $6(4-7)$ \\
\hline PrGA & $6(4-7)$ \\
\hline $\mathrm{ESR}, \mathrm{mm} / \mathrm{h}$ & $68(30-92)$ \\
\hline Elevated ESR* & $108(90)$ \\
\hline CRP, mg/l & $24(7-53)$ \\
\hline Elevated CRP** & $97(81)$ \\
\hline RF-positive rate & $83(69)$ \\
\hline ACPA-positive rate & $83(69)$ \\
\hline DAS28-CRP & $5.9(4.7-6.9)$ \\
\hline SDAI & $29(18-42)$ \\
\hline CDAI & $26(16-36)$ \\
\hline HAQ score & $1(0-2)$ \\
\hline \multicolumn{2}{|l|}{ Radiographic assessment } \\
\hline mTSS & $36(15-66)$ \\
\hline Joint space narrowing subscore & $5(1-20)$ \\
\hline Erosion subscore & $26(11-43)$ \\
\hline Bony erosions & $116(97)$ \\
\hline Radiographic joint damage, mTSS $>10$ & $95(79)$ \\
\hline \multicolumn{2}{|l|}{ Previous medications } \\
\hline DMARD- and corticosteroid-naive & $70(58)$ \\
\hline Low-dose glucocorticoid alone & $14(12)$ \\
\hline Low-dose glucocorticoid + DMARD & $25(21)$ \\
\hline DMARD alone & $11(9)$ \\
\hline
\end{tabular}

* Elevated ESR: > $15 \mathrm{~mm} / \mathrm{h}$ for males and $>20 \mathrm{~mm} / \mathrm{h}$ for females. ** Elevated CRP: CRP > $5 \mathrm{mg} / \mathrm{l}$. IQR: interquartile range; 28-TJC: 28-joint tender joint count; 28-SJC: 28-joint swollen joint count; PtGA: patient's global assessment of disease activity; PrGA: provider global assessment of disease activity; HAQ: Health Assessment Questionnaire; ESR: erythrocyte sedimentation rate; CRP: C-reactive protein; RF: rheumatoid factor; ACPA: anticyclic citrullinated peptide antibody; DAS28: 28-joint count Disease Activity Score; SDAI: Simplified Disease Activity Index; CDAI: Clinical Disease Activity Index; mTSS: modified total Sharp/van der Heijde score; DMARD: disease-modifying antirheumatic drug; RA: rheumatoid arthritis.

any disease-modifying antirheumatic drugs (DMARD) or glucocorticoids before recruitment. Thirty-nine (33\%) took low-dose glucocorticoids (equivalent to prednisone $\leq 10$ $\mathrm{mg} / \mathrm{d})$ alone $(\mathrm{n}=14)$ or with DMARD $(\mathrm{n}=15)$. Eleven $(9 \%)$ took DMARD without glucocorticoids. DMARD, including methotrexate $(n=30,25 \%)$, leflunomide $(n=18,15 \%)$, salazosulfadimidine $(\mathrm{n}=6,5 \%)$, and hydroxychloroquine $(\mathrm{n}=7,6 \%)$. None of them took biologic DMARD.

The median disease duration was 48 months (range $3-360)$. Thirty-three (28\%) patients had early RA $(<1 \mathrm{yr})$. Another $28 \%$ of patients $(\mathrm{n}=34)$ had late-stage RA $(>10$ yrs), and 53 (44\%) patients had mid-stage RA (1-10 yrs).

MRI features and RAMRIS of bilateral hands. The mean imaging time for the entire MRI examination of bilateral hands, including patient positioning and contrast agent injection, was $23 \pm 5 \mathrm{~min}$. Figure 1 showed typical MRI images in 2 patients with RA, 1 with early RA and the other with mid-stage RA. Among 240 hands of 120 patients with $\mathrm{RA}$, the occurrence frequencies of each MRI feature in wrist joints or bones were higher than those in MCPJ2-5 (Table 2 and Figure 2). Among all bones of bilateral hands, the top 3 bones with MRI osteitis were triquetrum (76\%), lunate (76\%), and scaphoid (74\%); the top 3 bones with MRI bone erosion were triquetrum, lunate, and capitate (all 93\%). The distribution of MRI synovitis was nearly consistent among patients in different stages of RA (Figure 3). Trapezoid and fifth metacarpal base showed more osteitis in mid-stage RA than in early RA. Wrist bones and MCPJ2 proximal showed bone erosion in early RA. The second to fifth metacarpal bases and the second to fourth MCPJ distal showed more bone erosion in mid-stage or late-stage RA (Figure 3).

The mean RAMRIS time for bilateral hands was $10 \pm 2$ min compared to unilateral hand of $7 \pm 2 \mathrm{~min}$. The median RAMRIS of synovitis, osteitis, and bone erosion were, respectively, 18 (IQR 10-26), 34 (11-52), and 58 (18-126). For early RA, median RAMRIS were 14 (10-23), 18 (8-36), and 29 (15-38), respectively. For mid-stage RA, median RAMRIS were 20 (11-30), 45 (16-61), and 90 (21-134), respectively. For late-stage RA, median RAMRIS were 21 (9-31), 36 (14-43), and 121 (40-196), respectively. Among the 3 groups, significant differences were seen in osteitis $(\mathrm{p}=0.036$; mid-stage RA $>$ early RA, $\mathrm{p}=0.018)$, and bone erosion ( $p<0.001$; mid-stage RA $>$ early RA, $p=0.001$; late-stage RA $>$ early RA, $\mathrm{p}<0.001$ ).

Further analyses of RAMRIS with clinical assessment showed significant correlation of synovitis score with DAS28-CRP $(r=0.559, \mathrm{p}<0.001)$, Simplified Disease Activity Index (SDAI, $r=0.572, p<0.001$ ), Clinical Disease Activity Index (CDAI, $r=0.536, p<0.001), 28$-joint swollen joint count (SJC28, $\mathrm{r}=0.467, \mathrm{p}<0.001$ ), 28-joint tender joint count (TJC28, $\mathrm{r}=0.460, \mathrm{p}<0.001)$, CRP $(\mathrm{r}=0.501$, $\mathrm{p}<0.001$ ), or erythrocyte sedimentation rate (ESR, $r=0.449$, $p<0.001)$. RAMRIS bone erosion score was significantly correlated with mTSS $(r=0.721, p<0.001)$ or erosion subscore of radiographic assessment $(\mathrm{r}=0.719, \mathrm{p}<0.001)$.

Comparison of MRI of bilateral hands with MRI of dominant unilateral hand. MRI images of bilateral hands showed asymmetrical involvement of bilateral hands in certain patients with RA (Figure 1D, Figure 1E, Figure 1F), indicating the possibility of misdiagnosis in the case of MRI of unilateral hands. Dominant hand was recommended for MRI of unilateral hands by OMERACT ${ }^{7,8}$. As shown in Table 2, when MRI of the dominant unilateral hand was analyzed, MRI synovitis in 5\% of wrists and 5-11\% of MCPJ2-5, MRI osteitis in 5\% of wrists, and 9-12\% of MCPJ2-5, and MRI bone erosion in $8-14 \%$ of MCPJ2-5 were misdiagnosed (McNemar test, all $\mathrm{p}<0.05)$. Similarly, when MRI of nondominant unilateral hand was analyzed, MRI synovitis in $12-19 \%$ of MCPJ2-5, MRI osteitis in 5\% of wrists and Personal non-commercial use only. The Journal of Rheumatology Copyright (C) 2018. All rights reserved. 

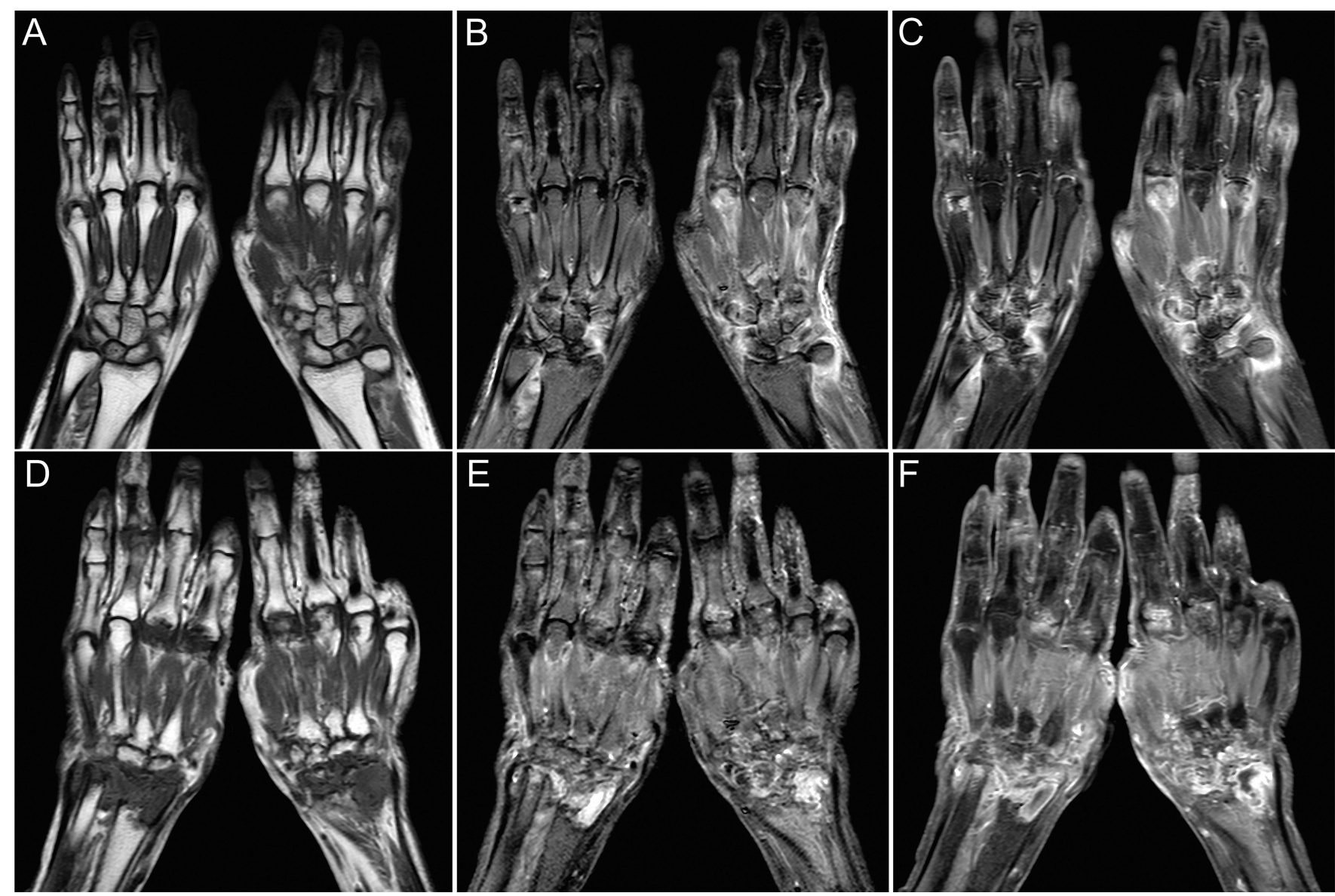

Figure 1. Typical MRI images of bilateral hands in 2 patients with RA. One was a 44-year-old woman and diagnosed with early RA (7 mos disease duration) Marked synovitis, osteitis, and bone erosion were shown in MRI (A-C). The other patient was a 64-year-old woman with 84 months of disease duration. Synovitis, osteitis, and bone erosion were shown as more severe in the nondominant hand versus the dominant hand (D-F). A and D. Coronal spin echo T1-weighted imaging. B and E. Coronal turbo spin echo fat-suppressed T2-weighted imaging. C and F. Contrast-enhanced coronal spin echo fat-suppressed T1-weighted imaging. MRI: magnetic resonance imaging; RA: rheumatoid arthritis.

$12 \%-18 \%$ of MCPJ $2-5$, and MRI bone erosion in $12-19 \%$ of MCPJ2-5 were misdiagnosed (McNemar test, all $\mathrm{p}<0.05)$.

Scatter plots in Figure 4 (top panels) were drawn with $\mathrm{Y}$ values of RAMRIS in dominant hands, and $\mathrm{X}$ values of RAMRIS in nondominant hands. Linear regression analyses showed significant constants (all p < 0.01), and 95\% CI of regression coefficients (B values) did not cover 1.000. Specifically, the linear models were markedly different from a $\mathrm{Y}=\mathrm{X}$ linear model, indicating that dominant hands were different from nondominant hands and could not represent nondominant hands to evaluate RAMRIS. In fact, there were $41 \%, 47 \%, 39 \%$ of dominant hands, respectively, having lower RAMRIS than nondominant hands. A similar trend could be seen in patients with early RA (Supplementary Figure 1A, available from the authors on request).

Comparison of bilateral-hands MRI with selected unilateral-hand MRI. The clinically more severe hand was usually selected for MRI of the unilateral hand, according to patients' complaints and physical examination ${ }^{3,10-23}$. In our study, the right hands of 42 patients (35\%) and left hands of 28 patients (23\%) were selected for clinically more severe involvement, and dominant hands (right hands of 49 patients and left hand of 1 patient) of 50 patients (42\%) were selected for equal involvement of bilateral hands. Among 240 wrists, $98 \%$ showed MRI synovitis and 95\% showed MRI osteitis (Table 2 ), but only $24 \%$ had swelling and $46 \%$ had tenderness (Supplementary Figure 2, available from the authors on request). There were $46 \%$ of wrist synovitis and $45 \%$ of wrist osteitis that were not noticed by joint tenderness and/or swelling. Among MCPJ, MCPJ2 showed the highest TJC $(25 \%)$ and the highest SJC (23\%). There were $33 \%$ of MCPJ 2 synovitis and $20 \%$ of MCPJ2 osteitis not noticed by joint tenderness and/or swelling.

As shown in Table 2, when selected MRI of unilateral hands were analyzed, MRI synovitis in 5\% of wrists, $7-12 \%$ of MCPJ except for MCPJ4, MRI osteitis in $8-13 \%$ of MCPJ2-5, and MRI bone erosion in 11-15\% of MCPJ2-5 were misdiagnosed (McNemar test, all $\mathrm{p}<0.05)$. Similarly, when MRI of nonselected unilateral hands were analyzed, MRI Personal non-commercial use only. The Journal of Rheumatology Copyright (C) 2018. All rights reserved. 
Table 2A. The occurrence frequencies of MRI features per joint, comparing dominant versus nondominant hand. Values are \% unless otherwise specified.

\begin{tabular}{|c|c|c|c|c|c|c|c|c|}
\hline \multirow[t]{3}{*}{ MRI Joint } & \multirow[t]{3}{*}{ Bilateral Hands } & \multirow{2}{*}{\multicolumn{3}{|c|}{ Unilateral Hand }} & \multicolumn{4}{|c|}{ Misdiagnosis by MRI } \\
\hline & & & & & \multicolumn{2}{|c|}{ Unilateral Dominant Hand } & \multicolumn{2}{|c|}{ Unilateral Nondominant Hand } \\
\hline & & Dominant & Nondominant & $\mathrm{p}^{*}$ & Rate & $\mathrm{p}^{*}$ & Rate & $\mathrm{p}^{*}$ \\
\hline Wrist & 98 & 93 & 94 & 1 & 5 & 0.031 & 4 & 0.063 \\
\hline MCPJ2 & 70 & 59 & 58 & 1 & 11 & $<0.001$ & 12 & $<0.001$ \\
\hline MCPJ3 & 62 & 52 & 46 & 0.281 & 10 & $<0.001$ & 16 & $<0.001$ \\
\hline \multicolumn{9}{|l|}{ Osteitis } \\
\hline Wrist & 95 & 90 & 90 & 1 & 5 & 0.031 & 5 & 0.031 \\
\hline MCPJ2 & 53 & 44 & 35 & 0.071 & 9 & $<0.001$ & 18 & $<0.001$ \\
\hline MCPJ3 & 48 & 38 & 33 & 0.362 & 10 & $<0.001$ & 15 & $<0.001$ \\
\hline MCPJ4 & 44 & 33 & 32 & 0.851 & 11 & $<0.001$ & 12 & $<0.001$ \\
\hline MCPJ5 & 55 & 43 & 42 & 0.856 & 12 & $<0.001$ & 13 & $<0.001$ \\
\hline MCPJ4 & 33 & 25 & 18 & 0.122 & 8 & 0.002 & 15 & $<0.001$ \\
\hline MCPJ5 & 49 & 38 & 32 & 0.311 & 11 & $<0.001$ & 17 & $<0.001$ \\
\hline
\end{tabular}

Table 2B. The occurrence frequencies of MRI features per joint, comparing selected versus nonselected hand. Values are \% unless otherwise specified.

\begin{tabular}{|c|c|c|c|c|c|c|c|}
\hline \multirow[t]{2}{*}{ MRI Joint } & \multicolumn{3}{|c|}{ Unilateral Hand } & \multicolumn{2}{|c|}{$\begin{array}{l}\text { Misdiagnosis by MRI of } \\
\text { Unilateral Selected Hand }\end{array}$} & \multicolumn{2}{|c|}{$\begin{array}{l}\text { Missed Diagnosis by MRI of } \\
\text { Unilateral Nonselected Hand }\end{array}$} \\
\hline & Selected & Nonselected & $\mathrm{p}^{*}$ & Rate & $\mathrm{p}^{*}$ & Rate & $\mathrm{p}^{*}$ \\
\hline Wrist $^{\dagger}$ & 93 & 94 & 1 & 5 & 0.031 & 4 & 0.063 \\
\hline MCPJ2 & 58 & 60 & 0.701 & 12 & $<0.001$ & 10 & $<0.001$ \\
\hline MCPJ3 & 53 & 44 & 0.071 & 9 & $<0.001$ & 18 & $<0.001$ \\
\hline \multicolumn{8}{|l|}{ Osteitis } \\
\hline Wrist $^{\ominus}$ & 91 & 89 & 0.774 & 4 & 0.063 & 6 & 0.016 \\
\hline MCPJ $2^{\wedge}$ & 40 & 38 & 1 & 13 & $<0.001$ & 15 & $<0.001$ \\
\hline $\mathrm{MCPJ}^{\wedge}$ & 35 & 35 & 1 & 13 & $<0.001$ & 13 & $<0.001$ \\
\hline MCPJ4^ & 36 & 29 & 0.185 & 8 & 0.002 & 15 & $<0.001$ \\
\hline $\mathrm{MCPJ}^{\wedge}$ & 44 & 41 & 0.585 & 11 & $<0.001$ & 14 & $<0.001$ \\
\hline MCPJ $4^{\wedge}$ & 21 & 22 & 1 & 12 & $<0.001$ & 11 & $<0.001$ \\
\hline MCPJ5 $5^{\wedge}$ & 38 & 31 & 0.175 & 11 & $<0.001$ & 18 & $<0.001$ \\
\hline
\end{tabular}

* P value was generated from McNemar test for comparing occurrence frequencies of each MRI feature per joint between dominant and nondominant hand, between selected and nonselected hand, and between MRI of bilateral hands and unilateral hand (dominant, nondominant, selected, or nonselected hand) considered as paired groups. P values in bold face are statistically significant. ${ }^{\dagger}$ Distal radioulnar joint, radiocarpal joint, and intercarpal-carpometacarpal joints were evaluated. ${ }^{\diamond} 15$ wrist bones were evaluated. ${ }^{\wedge}$ Metacarpal heads (proximal) and phalangeal bases (distal) were evaluated. MRI: magnetic resonance imaging; MCPJ: metacarpophalangeal joints.

synovitis in $10-21 \%$ of MCPJ2-5, MRI osteitis in $6 \%$ of wrists and $13-15 \%$ of MCPJ2-5, and MRI bone erosion in $10-18 \%$ of MCPJ2-5 were misdiagnosed (McNemar test, all p < 0.05).

Scatter plots in Figure 4 (bottom panels) were drawn with $\mathrm{Y}$ values of RAMRIS in selected hands and $\mathrm{X}$ values of RAMRIS in nonselected hands. Three linear models showed significant constants (all $\mathrm{p}<0.01$ ), and 95\% CI of regression coefficients (B values) did not cover 1.000. Specifically, the linear models were markedly different from a $\mathrm{Y}=\mathrm{X}$ linear model, indicating that selected hands were different from nonselected hands and could not represent nonselected hands to evaluate RAMRIS. There were $37 \%, 48 \%$, and $44 \%$ of

Personal non-commercial use only. The Journal of Rheumatology Copyright (c) 2018. All rights reserved. 

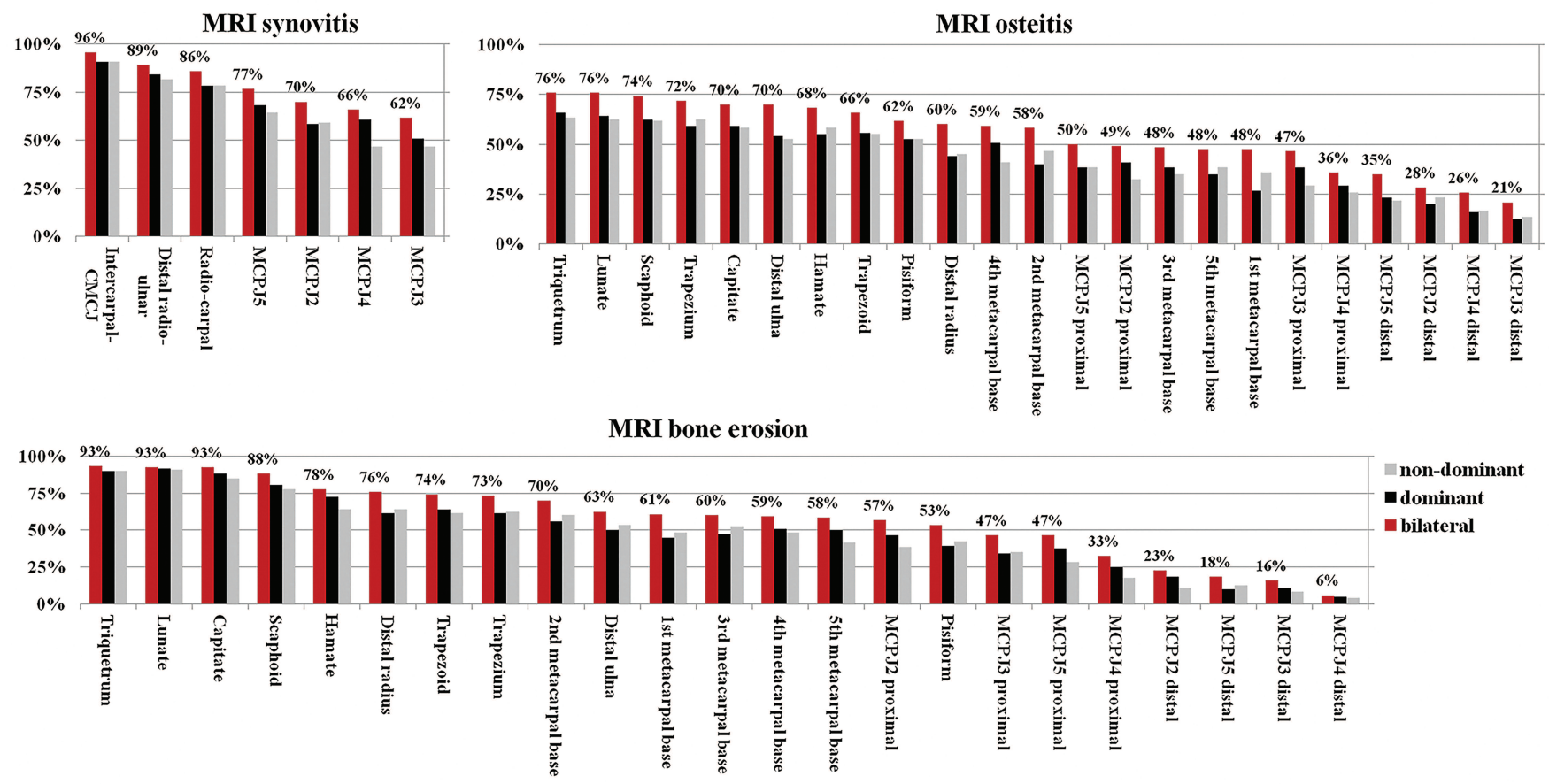

Figure 2. Distributions of MRI features in bilateral hands, dominant hands, and nondominant hands among 120 patients with RA. For MRI synovitis in wrist, the distal radioulnar joint, radiocarpal joint, and intercarpal CMCJ were evaluated. For MRI osteitis and bone erosion in wrist, 15 wrist bones were evaluated. For MRI osteitis and bone erosion in MCPJ, metacarpal heads (proximal) and phalangeal bases (distal) were evaluated. MRI: magnetic resonance imaging; CMCJ: carpometacarpal joints; MCPJ: metacarpophalangeal joints.
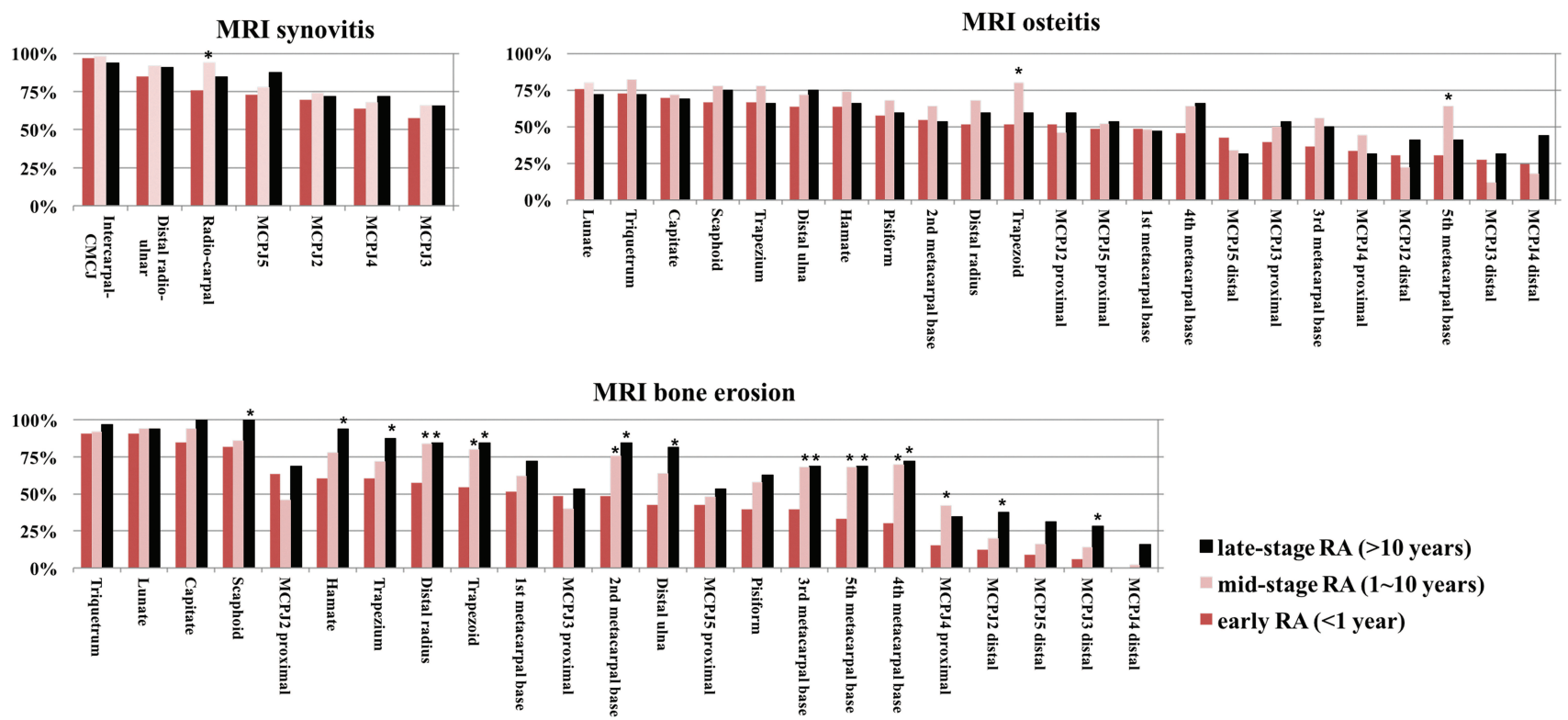

Figure 3. Distributions of MRI features per joint or bone among patients with early RA ( $<1 \mathrm{yr}, \mathrm{n}=33)$, mid-stage RA (1-10 yrs, $\mathrm{n}=53)$, and late-stage RA ( $>$ $10 \mathrm{yrs}, \mathrm{n}=34$ ) based on bilateral-hands MRI. RA: rheumatoid arthritis; MRI: magnetic resonance imaging; CMCJ: carpometacarpal joints; MCPJ: metacarpophalangeal joints. 


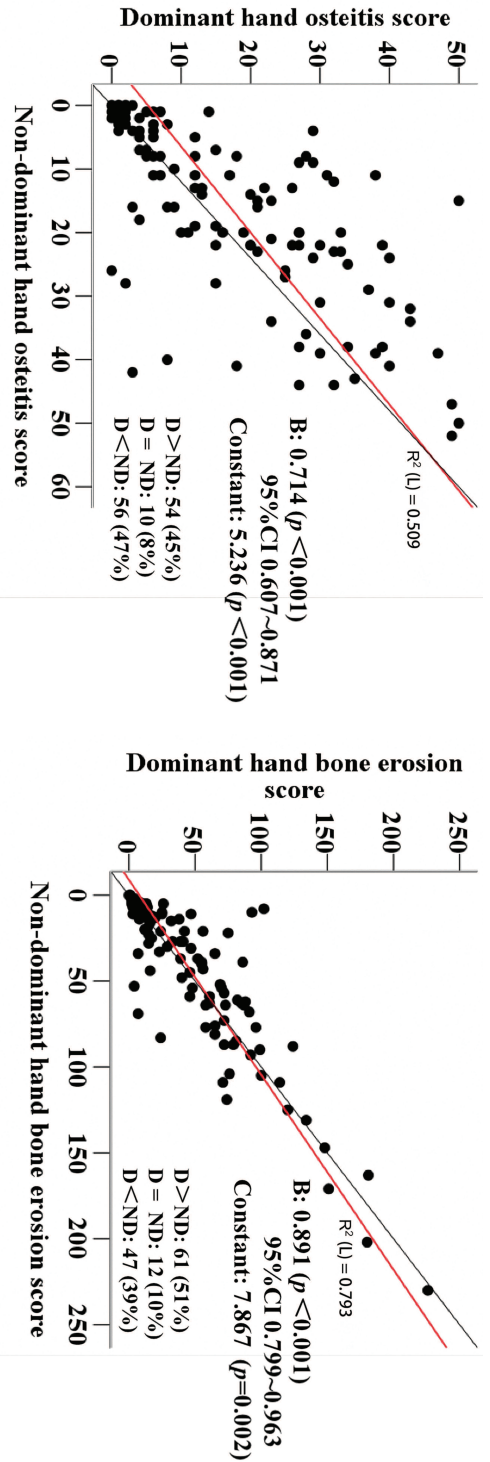

Dominant hand bone erosion
Figure 4. Scatter plots of RAMRIS in dominant and nondominant hands (top panels) and selected and nonselected hands (bottom panels). The black lines indicate $\mathrm{Y}=$ $\mathrm{X}$ linear model. The red lines indicate the real linear model by linear regression analyses for each scattered plot, of which $\mathrm{R}^{2}$, constant, and regression coefficient (B) and its 95\% CI are shown. D: dominant; ND: non-dominant; S: selected; NS: nonselected; RAMRIS: rheumatoid arthritis magnetic resonance image scoring.

\section{Personal non-commercial use only. The Journal of Rheumatology Copyright ()$^{2018}$. All rights reserved.}


selected hands, respectively, having lower RAMRIS than nonselected hands. A similar trend could be seen in patients with early RA (Supplementary Figure 1B, available from the authors on request).

\section{DISCUSSION}

In our study, bilateral hands of 120 consecutive patients with RA were assessed by high-field ( $3.0 \mathrm{~T}$ ) whole-body MRI with an 8-channel sense head coil, which took 23 min for imaging and $10 \mathrm{~min}$ for scoring. First, we reported the distribution and occurrence frequencies of MRI synovitis, osteitis, and bone erosion in different stages of RA resulting from complete sequences of MRI of bilateral hands. A strength of our study is the detailed comparison of MRI features and RAMRIS between MRI of bilateral hands and MRI of dominant or selected (clinically more severe) unilateral hands. We also reported on the significant correlation of RAMRIS synovitis score of bilateral hands with major disease activity indicators, including DAS28-CRP, SDAI, CDAI, SJC28, TJC28, CRP, or ESR, and significant correlation of RAMRIS bilateral hands bone erosion score with mTSS or erosion subscore.

In most clinical situations, MRI of the unilateral hand has been performed using extremity MRI with $100-\mathrm{mm}$ coils ${ }^{15,16,17}$. Limited by $100-\mathrm{mm}$ FOV, all sequences need to be acquired separately for the wrist and $\mathrm{MCPJ}^{32}$, which doubles the imaging time (e.g., 25 min for unilateral wrist ${ }^{33}$ and $58 \mathrm{~min}$ for unilateral wrist and $\mathrm{MCPJ}^{15}$ ). More recently, knee coils that can provide sufficient FOV to cover the wrist and MCPJ have been used for MRI of the unilateral hand, greatly reducing the imaging time ${ }^{34}$.

It is generally assumed that separate MRI of the unilateral hand may have higher image quality than simultaneous MRI of bilateral hands. Thus we set up 4 major measures to guarantee high image quality in our study. First, sandbags were put on forearms to avoid movement and to ensure bilateral hands lay horizontally in the center of the coil. Second, the field strength was $3.0 \mathrm{~T}$, which could offer a higher signal-to-noise ratio ${ }^{23}$. Third, the matrix values were appropriate, considering resolution, imaging time, and motion artifacts. Coronal and axial short-tau inversion recovery imaging sequence was used to suppress fat, which could guarantee homogeneous fat saturation with bilateral hands. The numbers of acquisitions were 3 for T1-weighted imaging and 2 for T2-weighted imaging. All these improved image quality. Finally, the multichannel synergic coils provided suitable coverage to enable bilateral hands to be scanned simultaneously, ensure the center positioning of bilateral hands, and reduce magnetic field heterogeneity in 3.0 T MRI. There are other coils of whole-body MRI that can be used for bilateral hands, such as dedicated extremity coil, wrist coil, knee coil, or cardiac coil (except flex coil, which is suboptimal for 3.0 T MRI because of its small coverage) $)^{35}$. In addition, high-solution 3D scans can also be used in MRI of bilateral hands. There have been 2 major clinical trials in
RA: 1 was a randomized, controlled trial of denosumab ${ }^{36}$; the other was a recently abstracted trial of tocilizumab in ACR $2017^{37}$. Both these studies successfully imaged bilateral hands separately with protocols that included thin-section, 3-D gradient-echo sequences, providing higher resolution than that achieved in our study. However, this MRI scan protocol nearly triples or quadruples the imaging time and thus has a lot of challenges in clinical use.

The occurrence frequencies of each MRI feature based on MRI of bilateral hands in our study were higher than those based on MRI of unilateral hands in a review of 4 major clinical trials of $\mathrm{RA}^{38}$. The general distributions were similar, except for a few discrepancies. MCPJ2 showed the most frequent synovitis in a published study ${ }^{38}$, but MCPJ5 did in our current study. Trapezium was the seventh wrist bone for osteitis in a published study ${ }^{38}$, but it was the fourth in our study, perhaps because of osteitis in nondominant hands, which was higher than osteitis in dominant hands. Further analyses were performed among patients in different stages of RA and showed the distribution of MRI synovitis and osteitis was nearly consistent. Wrist bones and MCPJ2 proximal showed bone erosion in early RA. The second to fifth metacarpal bases and the second to fourth MCPJ distal showed more bone erosion in mid-stage or late-stage RA.

The dominant hand has been recommended for RAMRIS by OMERACT since 2003. One explanation is that RAMRIS originated from databases consisting of MRI images of dominant wrists and/or $\mathrm{MCPJ}^{7,8}$. Another explanation may be that the joints of dominant hands could be more heavily affected than the nondominant hand because of mechanical burden. The published studies on the comparison between dominant and nondominant hands were mainly based on radiographs ${ }^{24,35,39}$. A prospective study in 2013 showed that both mTSS at single points and radiographic progression over time in patients with RA were highly correlated between dominant and nondominant hands ${ }^{24}$. There was only 1 MRI study that enrolled 46 bilateral wrists (MCPJ not included) and showed no significant differences of baseline erosions and erosive progression between dominant and nondominant hands, but MRI synovitis or osteitis could not be evaluated because of lack of T2-weighted and contrast-enhanced images ${ }^{40}$. In our study, MRI images of bilateral hands showed that dominant hands were not always more severe than nondominant hands. There were $41 \%, 47 \%$, and $39 \%$ of dominant hands, respectively, having lower RAMRIS than nondominant hands. When MRI of dominant unilateral hands were analyzed, MRI synovitis in 5\% of wrists and $5-11 \%$ of MCPJ2-5, MRI osteitis in 5\% of wrists and $9-12 \%$ of MCPJ2-5, and MRI bone erosion in $8-14 \%$ of MCPJ2-5 were misdiagnosed. Further linear regression analyses showed dominant hands could not represent nondominant hands to evaluate RAMRIS.

The clinically more severe hand was also usually selected for MRI of unilateral hands according to patients' complaints

Personal non-commercial use only. The Journal of Rheumatology Copyright @ 2018. All rights reserved. 
and physical examination ${ }^{3,10-23}$. MRI synovitis and osteitis were reported in clinically noninflamed joints ${ }^{3}$. In our study, we found that $46 \%$ of wrist synovitis, $29-52 \%$ of MCPJ2-5 synovitis, and $45 \%$ of wrist osteitis, $20-34 \%$ of MCPJ2-5 osteitis were not noticed by joint tenderness and/or swelling. Because of the poor concordance between physical examination and MRI, MRI synovitis in 5\% of wrists, $7-12 \%$ of MCPJ except for MCPJ4, MRI osteitis in $8-13 \%$ of MCPJ2-5, and MRI bone erosion in 11-15\% of MCPJ2-5 were misdiagnosed in the case of selected MRI of unilateral hands. Further linear regression analyses confirmed that clinically more severe hands could not represent the contralateral hands to evaluate RAMRIS.

There are 3 major limitations in this study. First, it was cross-sectional with only 1 timepoint MRI scan and it lacked prospective followup. The longitudinal behavior of putative erosions adds specificity to their diagnosis, because vascular channels and enthesis insertion sites do not change very much over time and can otherwise mimic erosions. Second, our study method is suboptimal because simultaneous MRI of bilateral hands and separate MRI of unilateral hands were not compared directly. However, it is difficult to do the contrast inspection twice because that may pose an ethical problem - another injection of contrast is harmful to the patient. Third, the most important advantage of MRI is for early RA, especially with normal radiographs, but there was only a small cohort of patients with early RA in our study. The comparisons between MRI of bilateral hands and MRI of unilateral hands need more data from patients with early RA. In addition, the interslice gap in axial view in our study is 2 $\mathrm{mm}$, which is suboptimal compared to other trials typically using contiguous slices with no gap. This limitation may diminish the sensitivity and specificity for bone erosions.

MRI of bilateral hands is more optimal than MRI of unilateral hands for RA, particularly with the improved performance of high-field whole-body MRI.

\section{ACKNOWLEDGMENT}

We thank all the patients and medical staff who generously contributed to this study. We thank Dr. Li Nan from Clinical Epidemiology Research Center, Peking University Third Hospital, who kindly provided statistical advice for this manuscript.

\section{REFERENCES}

1. Saraux A, Berthelot JM, Chalès G, Le Henaff C, Thorel JB, Hoang S, et al. Ability of the American College of Rheumatology 1987 criteria to predict rheumatoid arthritis in patients with early arthritis and classification of these patients two years later. Arthritis Rheum 2001;44:2485-91.

2. Colebatch AN, Edwards CJ, Østergaard M, van der Heijde D, Balint PV, D'Agostino MA, et al. EULAR recommendations for the use of imaging of the joints in the clinical management of rheumatoid arthritis. Ann Rheum Dis 2013;72:804-14.

3. Krabben A, Stomp W, Huizinga TW, van der Heijde D, Bloem JL, Reijnierse M, et al. Concordance between inflammation at physical examination and on MRI in patients with early arthritis. Ann Rheum Dis 2015;74:506-12.
4. van der Helm-van Mil AH. Imaging: use of MRI as an outcome measure in clinical trials in RA. Nat Rev Rheumatol 2012;8:643-4.

5. Østergaard M, Møller-Bisgaard S. Optimal use of MRI in clinical trials, clinical care and clinical registries of patients with rheumatoid arthritis. Clin Exp Rheumatol 2014;32:S-17-22.

6. Østergaard M, Peterfy C, Conaghan P, McQueen F, Bird P, Ejbjerg $\mathrm{B}$, et al. OMERACT rheumatoid arthritis magnetic resonance imaging studies. Core set of MRI acquisitions, joint pathology definitions, and the OMERACT RA-MRI scoring system. J Rheumatol 2003;30:1385-6.

7. Peterfy C, Edmonds J, Lassere M, Conaghan P, Østergaard M, McQueen F, et al. OMERACT Rheumatoid Arthritis MRI Studies Module. J Rheumatol 2003;30:1364-5.

8. McQueen F, Lassere M, Edmonds J, Conaghan P, Peterfy C, Bird P, et al. OMERACT Rheumatoid Arthritis Magnetic Resonance Imaging Studies. Summary of OMERACT 6 MR Imaging Module. J Rheumatol 2003;30:1387-92.

9. Østergaard M, Edmonds J, McQueen F, Peterfy C, Lassere M, Ejbjerg B, et al. An introduction to the EULAR-OMERACT rheumatoid arthritis MRI reference image atlas. Ann Rheum Dis Suppl 2005;64 Suppl 1:i3-7.

10. Ejbjerg BJ, Narvestad E, Jacobsen S, Thomsen HS, Østergaard M. Optimised, low-cost, low field dedicated extremity MRI is highly specific and sensitive for synovitis and bone erosions in rheumatoid arthritis wrist and finger joints: comparison with conventional high field MRI and radiography. Ann Rheum Dis 2005;64:1280-7.

11. Bird P, Ejbjerg B, Lassere M, Østergaard M, McQueen F, Peterfy C, et al. A multireader reliability study comparing conventional high-field magnetic resonance imaging with extremity low-field MRI in rheumatoid arthritis. J Rheumatol 2007;34:854-6.

12. Bensaoud N, Rostom S, Bahiri R, Hajjaj-Hassouni N. Efficacy of tocilizumab on MRI-determined bone oedema in rheumatoid arthritis. Clin Rheumatol 2015;34:1031-7.

13. Gandjbakhch F, Conaghan PG, Ejbjerg B, Haavardsholm EA, Foltz $\mathrm{V}$, Brown AK, et al. Synovitis and osteitis are very frequent in rheumatoid arthritis clinical remission: results from an MRI study of 294 patients in clinical remission or low disease activity state. J Rheumatol 2011;38:2039-44.

14. Baker JF, Ostergaard M, Emery P, Hsia EC, Lu J, Baker DG, et al. Early MRI measures independently predict 1-year and 2-year radiographic progression in rheumatoid arthritis: secondary analysis from a large clinical trial. Ann Rheum Dis 2014;73:1968-74.

15. Naraghi AM, White LM, Patel C, Tomlinson G, Keystone EC. Comparison of 1.0-T extremity MR and 1.5-T conventional high-field-strength MR in patients with rheumatoid arthritis. Radiology 2009;251:829-37.

16. Krabben A, Stomp W, van der Heijde DM, van Nies JA, Bloem JL, Huizinga TW, et al. MRI of hand and foot joints of patients with anticitrullinated peptide antibody positive arthralgia without clinical arthritis. Ann Rheum Dis 2013;72:1540-4.

17. Stomp W, Krabben A, van der Heijde D, Huizinga TW, Bloem JL, van der Helm-van Mil AH, et al. Are rheumatoid arthritis patients discernible from other early arthritis patients using $1.5 \mathrm{~T}$ extremity magnetic resonance imaging? A large cross-sectional study. J Rheumatol 2014;41:1630-7.

18. Krabben A, Stomp W, van Nies JA, Huizinga TW, van der Heijde D, Bloem JL, et al. MRI-detected subclinical joint inflammation is associated with radiographic progression. Ann Rheum Dis 2014;73:2034-7.

19. Orguc S, Tikiz C, Aslanalp Z, Erbay PD. Comparison of OMERACT-RAMRIS scores and computer-aided dynamic magnetic resonance imaging findings of hand and wrist as a measure of activity in rheumatoid arthritis. Rheumatol Int 2013;33:1837-44

20. Werner SG, Langer HE, Ohrndorf S, Bahner M, Schott P, Schwenke




$\mathrm{C}$, et al. Inflammation assessment in patients with arthritis using a novel in vivo fluorescence optical imaging technology. Ann Rheum Dis 2012;71:504-10.

21. MacIsaac KD, Baumgartner R, Kang J, Loboda A, Peterfy C, DiCarlo J, et al. Pre-treatment whole blood gene expression is associated with 14-week response assessed by dynamic contrast enhanced magnetic resonance imaging in infliximab-treated rheumatoid arthritis patients. PLoS One 2014;9:e113937.

22. Conaghan PG, Durez P, Alten RE, Burmester GR, Tak PP, Klareskog L, et al. Impact of intravenous abatacept on synovitis, osteitis and structural damage in patients with rheumatoid arthritis and an inadequate response to methotrexate: the ASSET randomised controlled trial. Ann Rheum Dis 2013;72:1287-94.

23. Schueller-Weidekamm C, Lodemann KP, Grisar J, Schueller G, Weber M, Kainberger F, et al. Contrast-enhanced MR imaging of hand and finger joints in patients with early rheumatoid arthritis: do we really need a full dose of gadobenate dimeglumine for assessing synovial enhancement at 3 T? Radiology 2013;268:161-9.

24. Knevel R, Kwok KY, de Rooy DP, Posthumus MD, Huizinga TW, Brouwer E, et al. Evaluating joint destruction in rheumatoid arthritis: is it necessary to radiograph both hands and feet? Ann Rheum Dis 2013;72:345-9.

25. Ejbjerg BJ, Vestergaard A, Jacobsen S, Thomsen HS, Østergaard M. The smallest detectable difference and sensitivity to change of magnetic resonance imaging and radiographic scoring of structural joint damage in rheumatoid arthritis finger, wrist, and toe joints: a comparison of the OMERACT rheumatoid arthritis magnetic resonance imaging score applied to different joint combinations and the Sharp/van der Heijde radiographic score. Arthritis Rheum 2005;52:2300-6.

26. Ma JD, Wei XN, Zheng DH, Mo YQ, Chen LF, Zhang X, et al. Continuously elevated serum matrix metalloproteinase-3 for 3-6 months predict one-year radiographic progression in rheumatoid arthritis: a prospective cohort study. Arthritis Res Ther 2015;17:289.

27. Mo YQ, Dai L, Zheng DH, Zhu LJ, Wei XN, Pessler F, et al. Synovial infiltration with CD79a-positive B cells, but not other B cell lineage markers, correlates with joint destruction in rheumatoid arthritis. J Rheumatol 2011;38:2301-8.

28. Baker JF, George M, Baker DG, Toedter G, Von Feldt JM, Leonard MB. Associations between body mass, radiographic joint damage, adipokines and risk factors for bone loss in rheumatoid arthritis. Rheumatology 2011;50:2100-7.

29. Conaghan P, Bird P, Ejbjerg B, O'Connor P, Peterfy C, McQueen F, et al. The EULAR-OMERACT rheumatoid arthritis MRI reference image atlas: the metacarpophalangeal joints. Ann Rheum Dis Suppl 2005;64 Suppl 1:i11-21.

30. Ejbjerg B, McQueen F, Lassere M, Haavardsholm E, Conaghan P, O'Connor P, et al. The EULAR-OMERACT rheumatoid arthritis
MRI reference image atlas: the wrist joint. Ann Rheum Dis Suppl 2005;64 Suppl 1:i23-47.

31. Eshed I, Krabbe S, Østergaard M, Bøyesen P, Møller JM, Therkildsen F, et al. Influence of field strength, coil type and image resolution on assessment of synovitis by unenhanced MRI-a comparison with contrast-enhanced MRI. Eur Radiol 2015; 25:1059-67.

32. Stomp W, Krabben A, van der Heijde D, Huizinga TW, Bloem JL, van der Helm-van Mil AH, et al. Aiming for a shorter rheumatoid arthritis MRI protocol: can contrast-enhanced MRI replace T2 for the detection of bone marrow oedema? Eur Radiol 2014; 24:2614-22.

33. Stomp W, Krabben A, van der Heijde D, Huizinga TW, Bloem JL, Østergaard M, et al. Aiming for a simpler early arthritis MRI protocol: can Gd contrast administration be eliminated? Eur Radiol 2015;25:1520-7.

34. Notohamiprodjo M, Glaser C, Horng A, Helck A, Bauner KU, Reiser MF, et al. Dynamic 3D-MR-angiography for assessing rheumatoid disease of the hand - a feasibility study. Eur J Radiol 2012;81:951-6.

35. Koh JH, Jung SM, Lee JJ, Kang KY, Kwok SK, Park SH, et al. Radiographic structural damage is worse in the dominant than the non-dominant hand in individuals with early rheumatoid arthritis. PLoS One 2015;10:e0135409.

36. Cohen SB, Dore RK, Lane NE, Ory PA, Peterfy CG, Sharp JT, et al; Denosumab Rheumatoid Arthritis Study Group. Denosumab treatment effects on structural damage, bone mineral density, and bone turnover in rheumatoid arthritis: a twelve-month, multicenter, randomized, double-blind, placebo-controlled, phase II clinical trial. Arthritis Rheum 2008;58:1299-309.

37. Peterfy C, Kremer J, Rigby WFC, Singer N, Birchwood C, Gill D, et al. MRI results following discontinuation of methotrexate in patients with rheumatoid arthritis treated with subcutaneous tocilizumab. Results from a randomized controlled trial [abstract 2962]. Arthritis Rheumatol 2017;69 Suppl 10:4218.

38. Peterfy CG, Countryman P, Gabriele A, Shaw T, Anisfeld A, Tsuji $\mathrm{W}$, et al. Magnetic resonance imaging in rheumatoid arthritis clinical trials: emerging patterns based on recent experience. J Rheumatol 2011;38:2023-30.

39. Yaku A, Hashimoto M, Furu M, Ito H, Yamakawa N, Yamamoto W, et al. Relationship between handedness and joint involvement in rheumatoid arthritis. Sci Rep 2016;6:39180.

40. Ostergaard M, Møller Døhn U, Duer-Jensen A, Hetland ML, Hørslev-Petersen K, Stengaard-Pedersen K, et al. Patterns of magnetic resonance imaging bone erosion in rheumatoid arthritis-which bones are most frequently involved and show the most change? J Rheumatol 2011;38:2014-7. 